\title{
Flux Monitoring Using the Torun 32-m Radio Telescope
}

\author{
J. Kijak \\ Institute of Astronomy, University of Zielona Góra, Poland \\ Y. Gupta \\ NCRA, TIFR, Pune University Campus, Pune, India \\ W. Lewandowski, G. Hrynek \\ TCfA, Nicolaus Copernicus University, Toruń, Poland \\ K. Krzeszowski \\ Institute of Astronomy, University of Zielona Góra, Poland
}

\begin{abstract}
We present flux density measurements for PSR B0329+54 at $4.8 \mathrm{GHz}$ obtained during one year.
\end{abstract}

\section{Observations and Results}

We have observed PSR B0329+54 during one year using the Torun 32-m radio telescope. Ten epochs of flux monitoring at $4.8 \mathrm{GHz}$ distributed over one year are reported here. Typical observations lasted about 24 hours in 3 minutes scans, but two epochs (Fig. 1) had longer observation times (5 and 2 days, respectively). The total intensity signal was obtained by summing the dualcircular polarization signals, and dedispersing over 64 channels covering the 192 $\mathrm{MHz}$ bandwidth. In order to convert to flux density we carried out regular calibration measurements using the injected signal of a noise diode, which was compared to the flux density of known continuum calibrators. The observations were interrupted regularly for calibration procedure and sometimes due to technical problems. We have obtained a combined time series of calibrated flux measurements for each epoch. Typical errors of flux measurements are about $10 \%$. The longer epochs of flux density time series are presented in Figure 1.

\section{Analysis and Conclusions}

For each epoch, the average flux density and modulation index were calculated. The average flux density $S_{\mathrm{av}}$ for the one year period is $16 \pm 6 \mathrm{mJy}$, which is consistent with the known spectrum of this pulsar (Maron et al. 2000). The modulation index $m$ varies between 0.7 and 1.3. To estimate the modulation timescale, we computed the structure functions for our data sets. The estimated timescale is 90 minutes, with an uncertainty of about 15 minutes. 


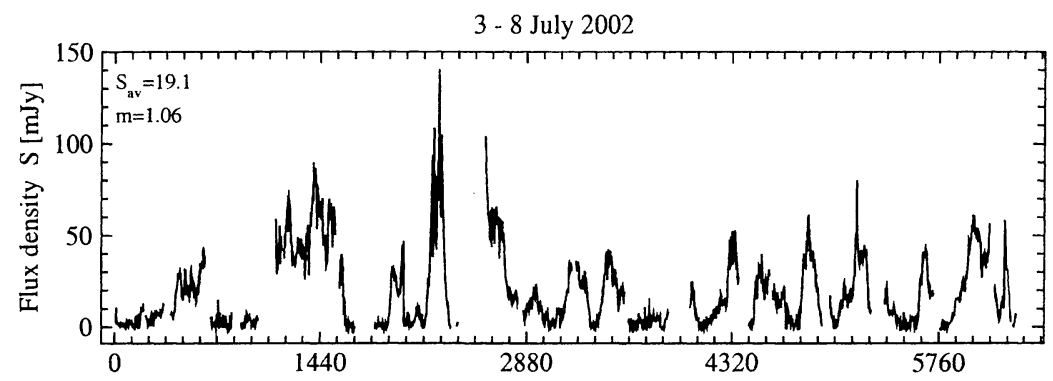

19 - 21 February 2003

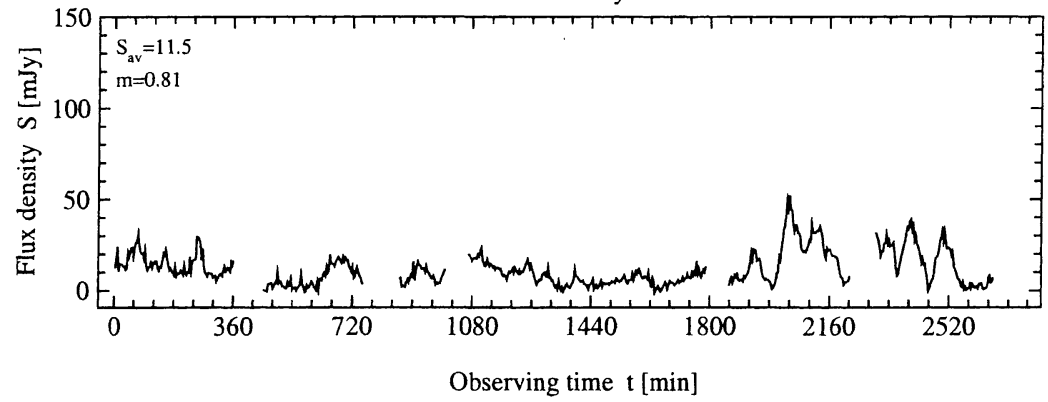

Figure 1. Two epochs of flux density time series. Note a significant difference in fluxes between these two epochs separated by about six months.

Summarizing, (1) we conclude from our preliminary results that the data quality is good enough for detailed studies of ISS of PSR B0329+54 at $5 \mathrm{GHz}$, (2) our analysis shows that the modulation index $m$ is close to unity, consistent with Malofeev et al. (1996) who reported $m \geq 0.5$. However, our time scale of $90 \mathrm{~min}$ is significantly larger than the 21 min given by Malofeev et al. (1996). Our result is much closer to the time scale of 80 min estimated by Kramer et al. (2003), (3) futher analysis of multi-epoch data spread over more than one year should result in better understanding of the scattering geometry for PSR $\mathrm{B} 0329+54$.

Acknowledgments. This work was supported in part by the Polish State Committee for Scientific Research Grant 2 P03D 008 19. JK acknowledges partial support from the IAU.

\section{References}

Kramer, M., Karastergiou, A., Gupta, Y., Johnston, S., Bhat, N. D. R., \& Lyne, A. G. 2003, A\&A, 407, 655

Malofeev, V. M., Shishov, V., Sieber, W., Jessner, A., Kramer, M., \& Wielebinski, R. 1996, A\&A, 308, 180

Maron, O., Kijak, J., Kramer, M., \& Wielebinski, R. 2000, A\&AS, 147, 195 